\title{
Persistent isolated unilateral hypoglossal nerve palsy
}

\author{
Austin Fletcher ${ }^{1}$ and Adam Fletcher ${ }^{2 *}$ \\ ${ }^{1}$ Saint James School of Medicine, Anguilla, Canada \\ ${ }^{2}$ York University, Toronto, Canada
}

\begin{abstract}
\section{Introduction}

The hypoglossal nerve plays an important role in speech and swallowing. Despite the nerves important function, damage to the nerve does not usually present with any functional loss or symptoms that would be overtly noticeable to most patients $[1,2]$. Patients may become aware of such lesions when brushing their teeth or when visiting a dentist. Causes of hypoglossal nerve palsy include intracranial space occupying lesions $(49 \%)$, trauma (12\%), stroke (6\%), hysteria $(6 \%)$, surgery (5\%), multiple sclerosis (5\%), infection (4\%), Guillian-Bare syndrome (4\%) and idiopathic causes (3\%) [3]. The patient in this case has persistent isolated unilateral left sided hypoglossal nerve palsy and cannot recall when the symptoms first started. The patient continues to experience the palsy and has not had the symptoms resolve or change. The patient has not experienced any challenges because of the symptoms.
\end{abstract}

Idiopathic Isolated unilateral hypoglossal nerve palsy is a rarely observed finding in clinical practice. Cases of persistent idiopathic isolated hypoglossal nerve palsy are extremely rare, one similar case documenting only one other documented case in the literature. Cases of this finding in patients with epilepsy further make this clinical finding a very rare entity. The presenting case is a 22 year old who complains of deviation of the tongue to the left side upon protrusion and atrophy of the left side of the tongue. The patient was diagnosed with epilepsy at age 10 and had been successfully controlled with medication remaining seizure free for over 10 years.

\section{Case report}

A 22-year-old patient was seen by a neurologist for a chief complaint of left sided tongue deviation upon protrusion. Fasciculations were also present on protrusion. There is also atrophy of the tongue on the left side. CN I-XI are intact, strength was $5 / 5$ in all muscle groups, sensation to sharp and dull were intact, DTR's were 2+ bilaterally, Babinski sign was negative, cerebellum exam was normal with a negative Romberg sign and the patient has a normal gait. The patient has no constitutional findings and stable vital signs.

The patient was diagnosed with epilepsy at age 10. At the time the patient experienced a total of 4 tonic-clonic seizures, and an unknown number of absence seizures. The patient was subsequently treated with valproic acid and has been seizure free for over 10 years. The patient has no history of head trauma, paralysis, paresis, paresthesia, weakness, and has no focal neurological signs. The patient has no history of meningitis or other infectious sequalae.

Multiplanar multisequence MR images were obtained of the skull base with and without gadolinium contrast on a 1.5 Tesla closed MR scanner (Figures 1-3).

The skull base appears symmetric with no obvious lesion identified.

The brainstem and visualized aspects of the brain parenchyma were within normal limits.
With contrast no abnormal enhancement was seen within the skull base.

\section{Discussion}

The hypoglossal nerve is the motor nerve responsible for movement of the tongue muscles playing an important role in articulation of speech [4]. Isolated hypoglossal nerve damage presents less frequently then isolated damage to cranial nerves III, IV, VI, and is a rare finding in clinical practice [5]. The motor distribution of the hypoglossal nerve is highly complex. The hypoglossal nerve has 4 associated sub nuclear components [6]. The hypoglossal nerve is commonly used in transplant surgery for facial paralysis, suggesting damage to the hypoglossal neve may present in subtle ways [6]. The hypoglossal nerve can be damaged anywhere from the medullary nucleus, hypoglossal canal or skull base [1]. Although the relative benign presentation of hypoglossal nerve palsy and its limited consequences for function, proper assessment and evaluation is necessary to rule out serious underlying causes of this rare pathology.

Ho et al. [2] identified a number of possible causes of hypoglossal

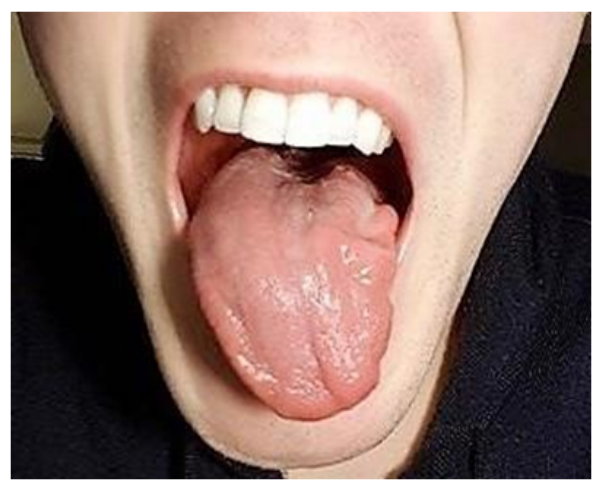

Figure 1. Tongue deviated to the left side.

Correspondence to: Adam Fletcher, Saint James School of Medicine, Canada, E-mail: agfletch@my.yorku.ca

Received: February 12, 2017; Accepted: February 25, 2017; Published: February 28,2017 


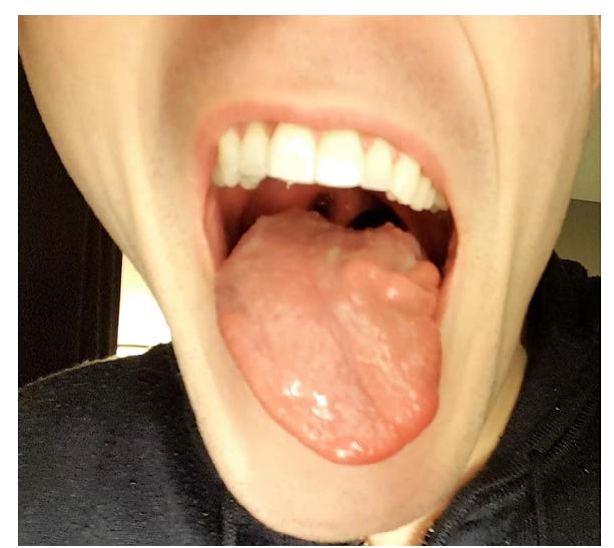

Figure 2. Atrophy of the left side of the tongue.

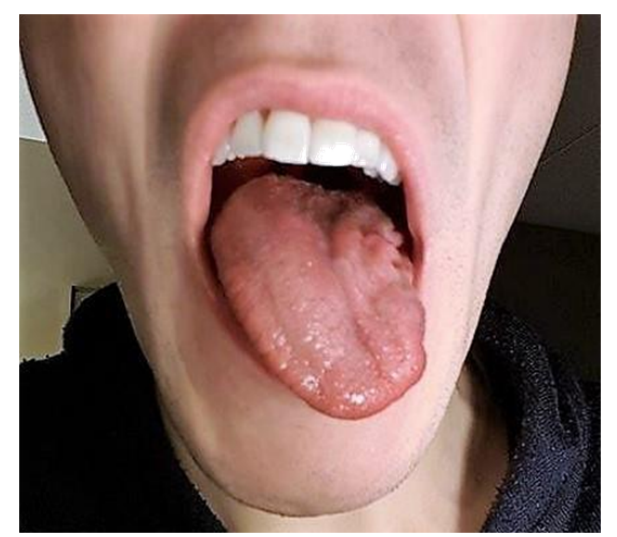

Figure 3. Atrophy of left side of tongue, fissures on left side of tongue. nerve palsy including; metastatic disease at the base of the skull, sarcoidosis, autoimmune disease, vasculitis, Arnold Chiari malformation, dural AV fistula of the transverse sinus, periostitis of the hypoglossal canal, post-retropharyngeal infection, surgical procedure near neck, acute polymyelitits, syringobulbia, thrombosis of the median branches of the vertebral artery, multiple sclerosis, carotid artery dissection or aneurysm, diabetes mellitus, lacunar infarct over the hypoglossal nucleus, complication of central venous catheterization, head and neck trauma, fracture through the occipital condyle, glomus tumor and meningioma.

\section{Conclusion}

This unique case of a patient who suffers from persistent isolated hypoglossal nerve palsy with epilepsy as a co-morbidity presents the case with a unique perspective. The clinical finding of persistent isolated hypoglossal nerve palsy itself is a rare clinical entity; made even more rare when this finding occurs in a patient with epilepsy. Further exploration into epilepsy as a possible cause of isolated hypoglossal nerve palsy should be made.

\section{References}

1. Ahmed SV, Akram MS (2014) Isolated unilateral idiopathic transient hypoglossal nerve palsy. BMJ Case Rep. [Crossref]

2. Ho MWS, Fardy MJ, Crean SJV (2004) Persistent idiopathic unilateral isolated hypoglossal nerve palsy: A case report. Br Dent $J$ 196: 205-207. [Crossref]

3. Keane JR (1996) Twelfth-Nerve PalsyAnalysis of 100 Cases. Arch Neurol 53: 561-566. [Crossref]

4. Mahadevappa K, Chacko T, Nair AK.Isolated unilateral hypoglossal nerve palsy due to vertebral artery dissection. Clin Med Res 10: 127-30. [Crossref]

5. Shikino K, Noda K, Ikusaka M (2017) Transient Idiopathic isolated unilateral Hypoglossal nerve palsy. J Gen Intern Med 28: 591.[Crossref]

6. Yoon JH, Cho KL, Lee HJ, Choi SH, Lee KY, et al. (2011) A case of idiopathic isolated hypoglossal nerve palsy in a Korean child. Korean J Pediatr 54: 515-517. [Crossref]

Copyright: ( 92017 Fletcher A. This is an open-access article distributed under the terms of the Creative Commons Attribution License, which permits unrestricted use, distribution, and reproduction in any medium, provided the original author and source are credited. 